The Astrophysical Journal, 477:722-731, 1997 March 10

(C) 1997. The American Astronomical Society. All rights reserved. Printed in U.S.A.

\title{
A SELF-CONSISTENT PHOTOIONIZATION-DUST CONTINUUM-MOLECULAR LINE TRANSFER MODEL OF NGC 7027
}

\author{
KeVIN VolK AND Sun KwoK \\ Department of Physics and Astronomy, University of Calgary, Calgary, Alberta, Canada T2N 1N4 \\ Received 1996 June 20; accepted 1996 September 23
}

\begin{abstract}
A model to simulate the entire spectrum $(1000 \AA$ to $1 \mathrm{~cm})$ of the high-excitation young planetary nebula NGC 7027 is presented. The ionized, dust, and molecular components of the object are modeled using geometric parameters obtained from visible, radio, infrared, and $\mathrm{CO}$ data. The physical processes considered include recombination lines of $\mathrm{H}$ and $\mathrm{He}$, collisional excited lines of metals, bf and $\mathrm{ff}$ continuum radiations, two-photon radiation, dust continuum radiation, and molecular rotational and vibrational transitions. The dust component is assumed to be heated by a combination of direct starlight and the line and continuum radiation from the ionized nebula. The molecular component of the nebula is coupled to the dust component through the stimulated absorption of the dust continuum radiation. Specifically, we compare the predicted fluxes of the CO rotational lines and the $179.5 \mu \mathrm{m}$ water rotational line to those observed by the Infrared Space Observatory satellite. From the observed ratio of these lines, we derived a gas phase water-to- $\mathrm{H}_{2}$ abundance ratio of $3.2 \times 10^{-7}$ assuming that the water is uniformly mixed with the CO.

Subject headings: dust, extinction - ISM: individual (NGC 7027) - ISM: molecules molecular processes - radiative transfer
\end{abstract}

\section{INTRODUCTION}

NGC 7027 is a planetary nebula that more than any other object, has been responsible for revolutionizing our understanding of the planetary nebula phenomenon. While it is relatively innocuous in its optical appearance, it was found to have a very large mid-infrared excess due to dust emission (Woolf 1969; Gillett, Merrill, \& Stein 1972). It is also one of the strongest thermal radio continuum sources in the sky, suggesting the presence of an ionized region of very high emission measure (Terzian, Balick, \& Bignell 1974). The detection of the $J=1 \rightarrow 0$ rotational line of CO in NGC 7027 by Mufson, Lyon, \& Marionni (1975) came as a surprise, for it was not expected that molecules could exist in or around an ionized environment. Since this detection, NGC 7027 has been found to be a rich molecular source, with many carbon-based molecules discovered in its massive (>1 $M_{\odot}$ ) neutral molecular envelope. Mapping of the molecular envelope by millimeter interferometry suggests that it is located outside of the small $\left(\sim 10^{\prime \prime}\right)$ ionized region (Bieging, Wilner, \& Thronson 1991). The dust envelope coexists with the molecular gas and is responsible for the large extinction $\left(A_{V} \sim 3 \mathrm{mag}\right)$ seen in the nebula. The central star is clearly hotter than average for planetary nebulae, with hydrogen and helium Zanstra temperatures of 500,000 and 430,000 K, respectively, having been suggested (see Table VII-6 of Pottasch 1984). However, direct imaging of the central star indicates a lower, but still high, temperature of $\sim 200,000-250,000 \mathrm{~K}$ (Jacoby 1988; Walton et al. 1989; Heap \& Hintzen 1990; Hubeny, Heap, \& Lanz 1994). The high central-star temperature suggests that the star must be massive. The rapid evolution of the massive central star, together with the high density of the ionized region, suggests that the nebula is very young, having left the asymptotic giant branch only $\sim 10^{3}$ yr ago.

Conventional circumstellar chemistry models assume that all of the available oxygen in a carbon-rich molecular region is bound in the $\mathrm{CO}$ molecule and that all the rest of the molecular chemistry proceeds with essentially no free oxygen present. Since the optical spectrum of NGC 7027 suggests a C-to-O ratio greater than 2, one expects only carbon-based molecules in the gas. The near-infrared spectrum of NGC 7027 is dominated by emission features commonly attributed to the polycyclic aromatic hydrocarbon (PAH) molecules (Gillett, Forrest, \& Merrill 1973), and in the mid-infrared there is no sign of the $9.7 \mu \mathrm{m}$ silicate feature commonly observed in oxygen-rich circumstellar envelopes. It is therefore safe to assume that the dust in NGC 7027 is carbon-based.

It was a surprise, therefore, when the Infrared Space Observatory (ISO) detected water in NGC 7027 (ISO press release 1996). The ISO Long Wavelength Spectrometer (LWS) spectrum from 157 to $192 \mu \mathrm{m}$ shows a nebular emission line due to $\mathrm{C}$ II at $157.7 \mu \mathrm{m}$, three $\mathrm{CO}$ rotational lines $(16 \rightarrow 15$ at $162.8 \mu \mathrm{m}, 15 \rightarrow 14$ at $173.6 \mu \mathrm{m}, 14 \rightarrow 13$ at 186.0 $\mu \mathrm{m})$, and the $179.5 \mu \mathrm{m}$ rotational line $\left(2_{12} \rightarrow 1_{01}\right)$ of orthowater. The observations of these highly excited CO lines by ISO therefore provide new constraints to the molecular model of NGC 7027, which previously has been based on the fitting of the lowest $\mathrm{CO}$ rotational lines. The expanded spectral coverage offered by ISO fills a previous gap between the infrared and the submillimeter observations, and provides for the first time the opportunity to create a comprehensive model of NGC 7027 covering the entire spectrum from the UV to radio.

The most recent effort in the modeling of NGC 7027 was made by Hoare, Roche, \& Clegg (1992), who took into account both the ionization and the dust components. In this paper, we present a model which includes the radiative interactions between the ionized, molecular, and dust components in NGC 7207. Furthermore, we have made use of the observed strength of the $179 \mu \mathrm{m}$ water line to derive a water abundance in NGC 7027.

\section{THE MODEL}

Planetary nebulae are complicated systems involving close radiative and dynamic interactions between the 
central star and the nebula (Kwok 1994). On the radiative side, the ionized gas component is heated by photoionization from absorbing the Lyman continuum photons from the central star, and cooled by collisional-excited line emissions. The dust component is heated by a combination of the visible continuum photons from the central star and the line photons (in particular $\mathrm{Ly} \alpha$ ) from the ionized gas component, and cooled by self-radiation. The molecular gas component is heated by gas collisions and absorption of near-infrared continuum photons, and cooled by molecular rotational emission. Since the three components are thermally coupled, an analysis of the radiation transfer problem requires simultaneous solutions to the photoionization, dust continuum transfer, and the molecular line transfer equations.

While this problem seems to be intractably complicated, certain approximations are possible. First, the ionized component is heated by the central star alone and is not affected by the neutral components. Second, since most of the dust radiation is emitted in the far-infrared where the nebula is optically thin, there is very little feedback of the dust emission into the other components. Similarly, the molecular gas emits only line radiation; although these lines can be optically thick because of self-absorption, they do not constitute a significant source of heating of the dust component. However, near-infrared emission from the dust can be absorbed by the molecular gas via vibrational excitations, and the molecular line transfer problem is dependent on the output of the dust transfer problem.

In this paper, we have set up the problem in the following way. First, the ionized gas component is treated independently. All the permitted lines with significant optical depths (e.g., the recombination lines of $\mathrm{H}$ and $\mathrm{He}$ ) have their line transfer problems solved using the escape probability method. The output of the photoionization model, including the fluxes of the recombination lines of hydrogen and helium, the collisional excitation lines of metals, the boundfree (bf) and free-free (ff) continua, and the stellar continuum, is fed to the dust continuum transfer problem as the input radiation field. There is a dust component (consisting of a mixture of graphite and PAH grains) that is intermixed with the ionized gas. A second dust component, made up of amorphous carbon, is assumed to be outside of the ionized component. The radiation transfer problem for the second dust component (the neutral wind region) is solved selfconsistently, producing the continuum fluxes $\left[J_{v}(r)\right]$ at every radius. The molecular gas is assumed to be mixed with this dust component, and the values of $J_{v}(r)$ are used as a source of background radiation available for stimulated absorption by the molecules. The equation of transfer and the equation of statistical equilibrium are then solved for the relevant molecules (in our case $\mathrm{CO}$ and $\mathrm{H}_{2} \mathrm{O}$ ) to produce the emergent fluxes in each of the rotational transitions. A flow chart illustrating the interactions between various components is given in Figure 1.

The density profile for the gas in the ionized and neutral regions has a three-component structure. The ionized region is assumed to be a shell of constant density and constant filling factor, the values of which are taken to be free parameters with some guidance from the forbidden-line analyses available in the literature. The remainder of the circumstellar shell is taken to consist of a high-density neutral shell of small angular width and a stellar wind region with the usual inverse-square density profile. The two components of the neutral gas have been deduced from CO maps and line profile analysis (see Jaminet et al. 1991). A schematic diagram of the geometry of the source structure is shown in Figure 2.

\subsection{The Photoionization Model}

The photoionization model was constructed using the CLOUDY photoionization code of G. Ferland (see Ferland 1996 for a description of CLOUDY). For nebular elemental abundances the values of Middlemass (1990) were used. For elements not included in Middlemass (1990) we adopted solar abundances corrected by the depletion factors for nearby elements in the periodic table. For example, $\mathrm{Cu}$ is depleted to the same extent as $\mathrm{Fe}, \mathrm{Na}$ is depleted to the same extent as $\mathrm{Mg}$, and so on. The nebula is assumed to be spherically symmetric, although it is known to have an ellipsoidal shape. The parameters of this uniform density shell (summarized in Table 1) are derived from a variety of

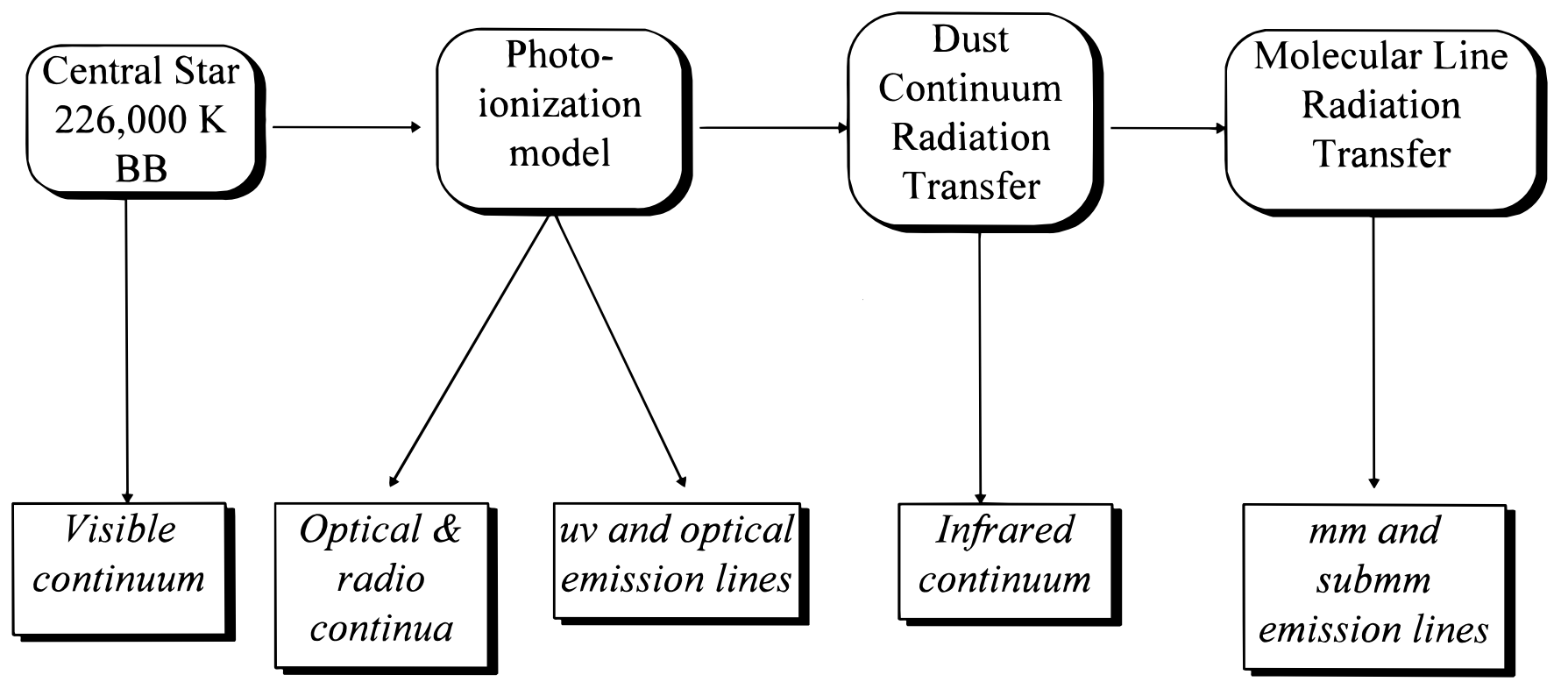

Fig. 1.-Flow chart of the model simulation 


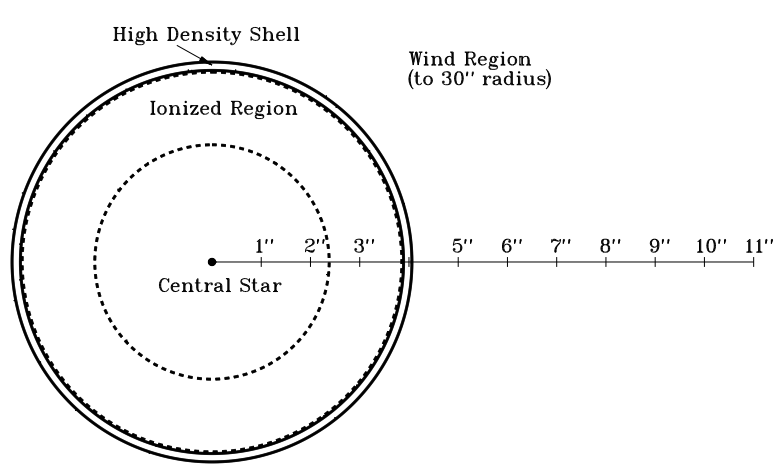

FIG. 2.-Schematic diagram of the geometric structure of NGC 7027

observed quantities: e.g., the density is deduced from observed forbidden-line ratios, the emission measure from the $\lambda=2 \mathrm{~mm}$ ff radio flux, and the angular size from the radio continuum map. The stellar temperature is taken to be equal to the hydrogen Zanstra temperature of Heap \& Hintzen (1990). The adopted distance of $930 \mathrm{pc}$ is consistent with the distance estimate of $0.94 \mathrm{kpc}$ of Masson (1986) and the revised value of $0.88 \pm 0.15 \mathrm{kpc}$ of Masson (1989). Because of the high temperature of the star, the ionized region does not have a sharp outer edge. While the photoionization model is calculated to a radius of $0.0224 \mathrm{pc}(4.96$ at $930 \mathrm{pc}$ ), the radius at which the ionization fraction of hydrogen is $50 \%$ is actually $0.0175 \mathrm{pc}\left(3{ }^{\prime \prime} 87\right.$ at $\left.930 \mathrm{pc}\right)$. The smaller radius was taken to be the effective radius of the ionized region.

The filling factor and density of the ionized region were adjusted to match the observed ratio of the inner and outer angular radii of the radio maps of Masson (1989), and to reproduce the observed $\mathrm{ff}$ radio continuum flux of the nebula. The best available for the ff emission flux density is $4.4 \pm 0.1 \mathrm{Jy}$ at $2000 \mu \mathrm{m}$ obtained at the James Clerk Maxwell Telescope (Sandell 1994). We were able to produce a model flux density at $2000 \mu \mathrm{m}$ of $4.37 \mathrm{Jy}$, which is very close to the observed value. Since NGC 7027 is optically thick at $5 \mathrm{GHz}$, the flux density at this frequency is not the best to use for fitting purposes. After including the optical depth effects, the model produces a $5 \mathrm{GHz}$ flux value of 5.11 $\mathrm{Jy}$, compared to the observed value of $5.7 \pm 0.6 \mathrm{Jy}$ (Scott 1973).

The CLOUDY photoionization model includes graphite dust grains at a default number abundance relative to hydrogen. The code calculates the dust temperature and

TABLE 1

MODEL PARAMETERS

\begin{tabular}{ll}
\hline \hline \multicolumn{1}{c}{ Parameter } & \multicolumn{1}{c}{ Value } \\
\hline Central star temperature $(\mathrm{K}) \ldots \ldots \ldots \ldots \ldots \ldots \ldots$ & 226,000 \\
Central star luminosity $\left(L_{\odot}\right) \ldots \ldots \ldots \ldots \ldots \ldots \ldots$ & 10,040 \\
Distance $(\mathrm{pc}) \ldots \ldots \ldots \ldots \ldots \ldots \ldots \ldots \ldots \ldots \ldots \ldots$ & 930 \\
Radius of ionized shell $(\mathrm{pc}) \ldots \ldots \ldots \ldots \ldots \ldots \ldots$ & 0.0107 (inner) \\
& $\ldots .0175$ (outer) \\
Electron density in ionized shell $\left(\mathrm{cm}^{-3}\right) \ldots \ldots \ldots$ & 80,000 \\
Nebular filling factor $\ldots \ldots \ldots \ldots \ldots \ldots \ldots \ldots \ldots$ & 0.5 \\
Mass of ionized shell $\left(M_{\odot}\right) \ldots \ldots \ldots \ldots \ldots \ldots \ldots$ & 0.022 \\
Radius of molecular shell $(\mathrm{pc}) \ldots \ldots \ldots \ldots \ldots \ldots$ & 0.0175 (inner) \\
& 0.0183 (outer) \\
Density of molecular shell $\left(\mathrm{cm}{ }^{-3}\right) \ldots \ldots \ldots \ldots \ldots$ & $9 \times 10^{5}$ \\
Mass of molecular shell $\left(M_{\odot}\right) \ldots \ldots \ldots \ldots \ldots \ldots \ldots$ & 0.196 \\
Mass of molecular wind $\left(M_{\odot}\right) \ldots \ldots \ldots \ldots \ldots \ldots$ & 1.65 \\
\hline
\end{tabular}

dust radiation field for each layer in the model without carrying out any radiative transfer calculations in the continuum. The emergent radiation field (including both line and continuum fluxes) from the CLOUDY model was used as input to the dust radiative transfer model discussed in the next section.

Since NGC 7027 shows strong emission features commonly attributed to the PAH molecules, we have added another dust component made up of PAH grains in the ionized gas. The cross sections for the PAH grains are based upon the analytical formulae given by Desert, Boulanger, \& Puget (1990). Some changes have been made in the discrete PAH feature strengths following the work of Schutte, Tielens, \& Allamandola (1993), who have better values for the bands near $11 \mu \mathrm{m}$. A plateau has also been added between the features based on the observed spectral continuum levels of Bregman et al. (1989). Since the equations from Desert et al. (1990) are only intended to apply to wavelengths longward of the Lyman limit, we have instituted a turnover (set at $607 \AA$ ) and a subsequent decrease of the cross section at wavelengths beyond the turnover. In addition, the sharp cutoff of the optical cross section at $8000 \AA$ in the paper of Desert et al. (1990) has been replaced by an exponential function beginning at $2500 \AA$. The opacity function is set to produce a feature-to-continuum ratio of 100 at $3.3 \mu \mathrm{m}$. This was done in part to avoid discontinuities in the opacity values and in part to allow the possibility of modeling the unusual red emission seen in a few reflection nebulae that have strong PAH emission. It should be noted that the long-wavelength opacity function of the PAH grains is poorly known, and therefore the infrared emission from the PAH grains is suspect aside from the discrete features. This is not a problem in practice, however, because the PAH grains are never observed in isolation; they are accompanied by other forms of dust that provide the continuum in between the emission features.

For the model, PAH clusters of radii 1.0 and $0.354 \mathrm{~nm}$ (120 and $15 \mathrm{C}$ atoms, respectively) were used. The smaller clusters are numerically twice as abundant as the larger clusters, but they carry about $25 \%$ of the mass of the larger clusters. The abundances were set using the formula from Schutte et al. (1993).

\subsection{The Dust Continuum Transfer Model}

NGC 7027 exhibits strong far-infrared emission (Telesco \& Harper 1977; McCarthy, Forrest, \& Houck 1978) which originates from a dust envelope outside of the ionized region (Kwok 1980). For modeling the dust in the neutral region outside the ionized region, a separate radiative transfer calculation was carried out using the DUSTCD radiative transfer code (Leung 1976). The dust was assumed to be amorphous carbon following the model of Hoare et al. (1992). The dust density distribution was chosen to match the derived $\mathrm{CO}$ mass-loss rate and radius range from Jaminet et al. (1991) with the addition of a high-density shell that was required to fit the higher $\mathrm{CO}$ rotational lines observed by ISO as described in the next section. The optical depth of the dust in this part of the shell was adjusted to produce a reasonable match to the observed dust continuum in the far infrared, particularly near $170 \mu \mathrm{m}$. The size of individual dust grains was set to be $0.1 \mu \mathrm{m}$. Although the model spectrum reasonably approximates the observed far-infrared continuum, it does not match the detailed spectral shape of NGC 7027. For example, the model dust spec- 
trum $\left(\lambda F_{\lambda}\right)$ peaks at $\sim 23 \mu \mathrm{m}$ rather than at $\sim 30 \mu \mathrm{m}$ as is observed. This disagreement is in part due to the omission of the $30 \mu \mathrm{m}$ feature in our opacity function (Forrest, Houck, \& McCarthy 1981).

The dust model was calculated with the nebular radiation field (output from CLOUDY) as the energy source at the inner radius and with the expected interstellar radiation field (the standard Habing radiation field and the cosmic background radiation field at long wavelengths) as an energy source at the outer radius. The model gives an optical depth of 0.0095 at $11.2 \mu \mathrm{m}$, and 1.47 at $5500 \AA$. The corresponding extinction at the $V$ band is about $40 \%$ lower than the observed optical extinction in NGC 7027. The derived dust-to-gas mass ratio is 0.00099 , which is slightly larger than the value found by Hoare et al. (1992). This represents a depletion of the dust by a factor of $3.1 \mathrm{com}$ pared to the default grain abundance we use for carbon star dust shell models.

The output radiation field $J_{v}$ as a function of radius derived in the radiative transfer model was used in the molecular line calculations described in the next section. The continuum from the dust model was used as the base continuum on which the spectral lines are seen when the far-infrared spectrum was simulated.

\subsection{The Molecular Line Transfer Model}

The first three rotational transitions of CO from NGC 7027 were analyzed by Jaminet et al. (1991). They derived the mass-loss rate, the $\mathrm{CO}$ abundance, and the kinetic temperature function by fitting the observed profiles. They found that the molecular gas does not form a spherically symmetric shell and derived a "pole"-to-" equator" ratio of 2.2 (from $1.0 \times 10^{-4}$ to $2.2 \times 10^{-4} M_{\odot} \mathrm{yr}^{-1}$ ) for the massloss rates. They also noted that there are slightly extended wings on the CO lines that imply that there is a region of higher speed outflow, which they attributed to a thin shell of higher density (and likely higher temperature) gas that exists just outside the ionized region and just inside the main part of the stellar wind shell. Adopting the parameters derived by Jaminet et al. (1991), we have the wind part of the circumstellar envelope extending from 0.0183 to 0.135 pc (assuming a distance of $0.93 \mathrm{kpc}$ ). In addition to this wind component, we have assumed the presence of a shell of constant density and gas temperature (see Table 1) between the ionized region and the start of the wind region. As will be shown later, this shell is needed to fit the strengths of the high rotational lines of $\mathrm{CO}$.

The remaining parameters (adopted from Jaminet et al. (1991)) are $M \sim 2.2 \times 10^{-4} M_{\odot} \mathrm{yr}^{-1}, V=15.2 \mathrm{~km} \mathrm{~s}^{-1}$, a $\mathrm{CO} / \mathrm{H}_{2}$ number abundance of $2 \times 10^{-4}$, a gas kinetic temperature $\left(T_{\text {kin }}\right)$ of $30 \mathrm{~K}$ at radius $0.03607 \mathrm{pc}$, and a radial dependence of $r^{-0.85}$ for $T_{\text {kin }}$. Using standard composition, this means that the $\mathrm{H}_{2}$ number density is $49080 \mathrm{~cm}^{-3}$ at the inner radius of the wind region. The density profile is assumed to be inverse-square over the range of radii in the wind region.

The molecular line transfer model is solved by obtaining simultaneous solutions to the statistical equilibrium equation and the radiation transfer equation in the standard escape probability formulation. Solutions were obtained independently for $\mathrm{CO}$ and for $\mathrm{H}_{2} \mathrm{O}$ using a combination of iterative and Newton-Raphson methods. For the $\mathrm{CO}$ model, the first 100 rotational levels in each of the first three vibrational states are included. For each of the ortho and para forms of water, the first three vibrational states and a total of 183 vibrational/rotational levels are included. The program adjusts the number of levels according to the level populations to eliminate upper levels with very small populations. The model calculations were done with 901 layers spaced logarithmically between the inner and outer radii. The radiation field from the dust radiation transfer calculation, which is tabulated as a function of radius and frequency, was used with the molecular level population equations. Once the level populations and local escape probabilities were determined as functions of radius for the desired lines, the line profiles were simulated for a particular distance and Gaussian beam size. For comparison with the ISO observations ( $(3)$, the "beam" was assumed to have a square profile rather than a Gaussian form.

The initial run of the CO level population program with the parameters from Jaminet et al. (1991) produced agreement with their results for the low-frequency rotational lines. However, the higher $J$ line strengths from the model were far too low. It was found to be necessary to have a constant-density, higher temperature shell at the base of the molecular wind in order to excite the higher CO lines. Model trials were made at various temperatures to reproduce the strengths of the observed CO lines. A high-density shell was found to be necessary, since the radiation field alone does not have a high enough intensity at wavelengths near $150 \mu \mathrm{m}$ to significantly populate the high- $J$ states. If these states are primarily populated by collisions, then the kinetic temperature (which will be close to the excitation temperature) has to be high enough to reproduce the observed brightness temperature of the lines. The shell parameters are also constrained by the creation of population inversion, since CO masers are not observed in NGC 7027 in the lower rotational lines. The thickness of the shell assumed in the model was adjusted to keep the total mass to, at most, about $10 \%$ of the total mass of gas in the neutral component. The goal was to produce a model with the proper CO lines which used the smallest possible shell mass.

\section{COMPARISON WITH THE ISO SPECTRUM OF NGC 7027}

The integrated strengths of the molecular lines expected in the ISO LWS region were calculated from the molecular transfer model and then added on top of the dust continuum assuming a Gaussian line shape with a FWHM of 0.70 $\mu \mathrm{m}$, which is approximately the line width of ISO. The C II $157.7 \mu \mathrm{m}$ emission line was added in the same way using its total flux from the photoionization model.

\subsection{The $\mathrm{CO}$ Lines}

The ISO LWS spectrum of NGC 7027 shows three CO rotational lines with approximately equal strengths (within the uncertainties of the uncalibrated spectrum). In order to reproduce these three high-level CO lines, a shell temperature of $220 \mathrm{~K}$ is needed. The shell molecular hydrogen density used was $900,000 \mathrm{~cm}^{-3}$, about 18 times the density in the wind at the interface with the shell and about 22 times the density in the ionized region allowing for the filling factor. This value could not be reduced more than a few percent without losing convergence in the model calculation due to population inversions. The inner radius of the shell in the model calculation was set to $0.0175 \mathrm{pc}$, giving a mass in the shell of $0.196 M_{\odot}$ to the outer radius of 0.0183 pc, compared to a mass of $1.65 M_{\odot}$ in the wind component. This model produced the higher $\mathrm{CO}$ rotational lines at the 
correct relative intensity and with about the proper strength compared to the model dust continuum.

In the model, the hot shell does not significantly change the strengths of the lower $\mathrm{CO}$ rotational lines because the escape probability values are small for these lines under the shell conditions and because of beam dilution effects. For the beam size used by Jaminet et al. (1991) (14" at CO $1 \rightarrow 0$ ), the hot shell contributes about $5 \%$ of the total emission according to the model. The value is higher for the $2 \rightarrow 1$ and $3 \rightarrow 2$ lines, but the dominant contribution still comes from the more extended wind region. In contrast, for the higher rotational lines, the contribution from the wind region is $\sim 6$ orders of magnitude smaller than that from the shell.

\subsection{The $\mathrm{C}$ II Line}

The strength of the $157.7 \mu \mathrm{m}$ [C II] line derived from the photoionization model is weaker than the line observed by ISO, by perhaps as much as a factor of 2 if there are no significant calibration effects across the wavelength range. As the ionization threshold for neutral carbon is less than that for hydrogen, a significant amount of the emission may be produced in the photodissociation region beyond the ionized region. Since we are not attempting detailed fits to all the nebular emission lines, we did not try to adjust the photoionization code results to give a perfect fit to the $\mathrm{C}$ II line.

\subsection{The $179.5 \mu \mathrm{m}$ Water Line}

The $179.5 \mu \mathrm{m}$ line comes from the $2_{12} \rightarrow 1_{01}$ transition of the ortho form of the water molecule. This transition is from the second excited rotational state to the ground rotational state of the molecule in the ground vibrational state. There is a transition at $556.945 \mathrm{GHz}(538.28 \mu \mathrm{m})$ from the first excited rotational state $\left(1_{10}\right)$ to the ground state but no allowed transition between the $2_{12}$ and $1_{10}$ levels. The 557 $\mathrm{GHz}$ transition cannot be observed from the ground, but will be a target of observation by the upcoming $S W A S$ and Odin missions. The ortho form of water is expected to be the dominant form in equilibrium conditions with a $3: 1$ abundance ratio. The actual abundance ratio will be sensitive to the formation conditions for the water molecules and the physical conditions of the gas. In our model the ortho- $\mathrm{H}_{2} \mathrm{O}$ abundance is a free parameter and the total $\mathrm{H}_{2} \mathrm{O}$ abundance is assumed to be $4 / 3$ of this value.

\subsubsection{Water Abundance}

We assumed the water and CO molecules to be uniformly mixed, and the molecular line transfer models were calculated for various $\mathrm{H}_{2} \mathrm{O} / \mathrm{CO}$ number ratios. At high water abundances (e.g., ortho- $\mathrm{H}_{2} \mathrm{O} / \mathrm{CO}>0.05$ ), the radiative transfer effects are more severe and the program sometimes failed to converge. At very high water abundances (e.g., ortho- $\mathrm{H}_{2} \mathrm{O} / \mathrm{CO}=1$ ), the water lines are so optically thick and the escape probability values so small that the spontaneous transitions are relatively unimportant. In this case, the level populations are dominated by the collisions. At very low abundances (e.g., ortho- $\mathrm{H}_{2} \mathrm{O} / \mathrm{CO}<0.01$ ), the level populations are controlled by the radiation field for the range of densities and temperatures considered here.

Models were generated to cover the ortho- $\mathrm{H}_{2} \mathrm{O} / \mathrm{CO}$ abundance ratio range between 0.0005 and 0.128 , in steps of multiples of 2 (Table 2). Later, when the more accurate 179.5 to $186.0 \mu \mathrm{m}$ line ratio $(=0.613)$ became available (Liu
TABLE 2

Model Line RATIOS FOR THE ORTHO-H $\mathrm{H}_{2} \mathrm{O} 2_{12} \rightarrow 1_{01}$ Line AT $179.5 \mu \mathrm{m}$ AND THE $186.0 \mu \mathrm{m}$ CO $14 \rightarrow 13$ ROTATIONAL Line as a Function of the Relative Abundance OF THE MOLECULES

\begin{tabular}{lc}
\hline \hline$\left[\right.$ Ortho- $\left.\mathrm{H}_{2} \mathrm{O} / \mathrm{CO}\right]$ & $179 \mu \mathrm{m} \mathrm{H}_{2} \mathrm{O} / 186 \mu \mathrm{m} \mathrm{CO}$ line ratio \\
\hline $0.0005 \ldots \ldots \ldots \ldots \ldots$ & 0.399 \\
$0.001 \ldots \ldots \ldots \ldots \ldots$. & 0.542 \\
$0.002 \ldots \ldots \ldots \ldots \ldots \ldots$ & 0.652 \\
$0.004 \ldots \ldots \ldots \ldots \ldots$. & 0.729 \\
$0.008 \ldots \ldots \ldots \ldots \ldots$. & 0.798 \\
$0.016 \ldots \ldots \ldots \ldots \ldots$. & 0.859 \\
$0.032 \ldots \ldots \ldots \ldots \ldots$. & $0.840^{\mathrm{a}}$ \\
$0.064 \ldots \ldots \ldots \ldots \ldots$. & $0.841^{\mathrm{a}}$ \\
$0.128 \ldots \ldots \ldots \ldots \ldots$ & 1.162 \\
\hline
\end{tabular}

${ }^{a}$ These entries are less accurate because of convergence problems.

et al. 1996), we carried out additional model runs to produce a close fit to the observed ratio. Using a power-law interpolation between the 0.001 and 0.002 cases, we were able to find an abundance ratio of $1.2 \times 10^{-3}$ which can produce a model line ratio within $1 \%$ of the observed 0.61 value. Assuming a $10 \%$ error in the observed line ratio, we estimate the uncertainty in the ortho- $\mathrm{H}_{2} \mathrm{O} / \mathrm{CO}$ abundance ratio to be $0.8 \times 10^{-3}$ to $1.7 \times 10^{-3}$.

At very high water abundances (about 30 times higher than our best-fit value), there are some convergence problems in the code due to population inversion created by stimulated absorption of the dust radiation field. We note that such population inversion problems do not arise if we substitute the dust radiation transfer by just the cosmic background radiation field. This difference underscores the importance of carrying out the calculations consistently for the nebular, dust shell, and molecular components.

\subsubsection{The Distribution of Water in NGC 7027}

In this model we have not considered the effects of photodissociation, and the molecular abundances are assumed to be constant throughout the nebula. However, it is possible that the water molecules exist mainly in the inner part of the neutral region, since water is more easily dissociated by ultraviolet radiation than is $\mathrm{CO}$. In order to assess the possible errors due to the neglect of chemical effects, we have examined the relative contributions of the various layers to the total strength of the $2_{12} \rightarrow 1_{01}$ line. The shell produces about $15 \%$ of the total emission for the best-fit abundance. This fraction increases with increasing water abundance until it flattens off at about $45 \%$ for the models with the largest abundances, where the line is $80 \%$ stronger than in the best-fit case. Such a high water abundance $\left(\left[\mathrm{H}_{2} \mathrm{O} / \mathrm{CO}\right]>0.2\right)$ is unlikely, for it implies that $\sim 5 \%$ of oxygen is in water. We therefore conclude that the water must be distributed over an extended region, overlapping with a significant fraction of the observed $\mathrm{CO}$ envelope.

An alternative explanation of the presence of the water line in NGC 7027 is to assume the existence of an oxygenrich envelope outside the carbon-rich nebula. In our model, between $30 \%$ and $40 \%$ of the total water line strength is produced beyond an angular radius of $15^{\prime \prime}$. Although this fraction will increase with higher water abundances, we were unable to produce the observed line strength even with the highest water abundance. This suggests that it is not possible for the water line to originate exclusively from the outer parts of the wind unless the $\mathrm{H}_{2} \mathrm{O} / \mathrm{CO}$ abundance ratio 


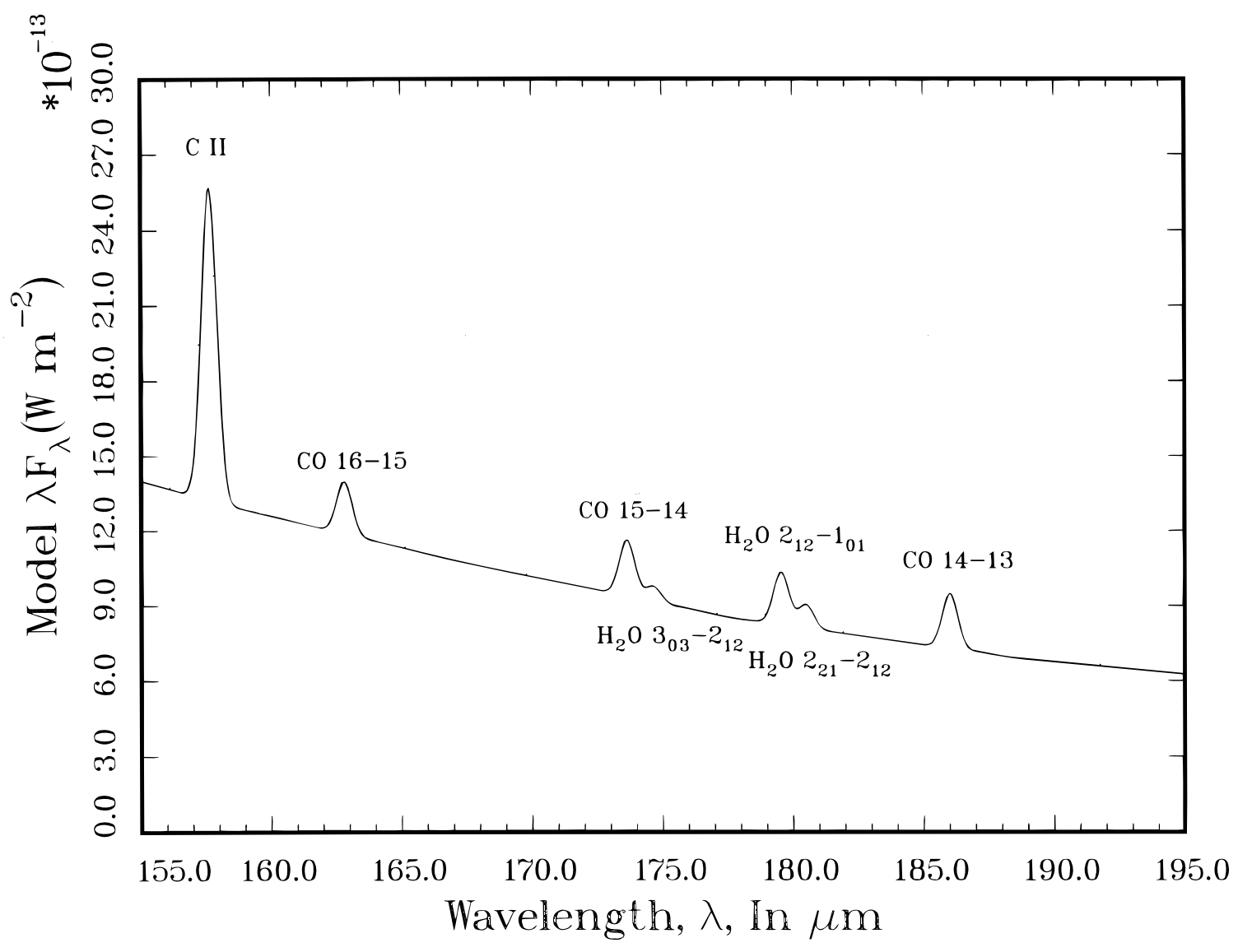

FIG. 3. - Simulated ISO LSW spectrum of NGC 7027 with an assumed water abundance of $3.2 \times 10^{-7}$

is very high. If this were the case, owing to the high optical depths and the very low escape probabilities, the excitation temperatures would be nearly equal to the kinetic temperature, which is quite low at large radii. If the water abundance is so high in the outer parts of the shell, then it would seem likely that dissociation of water to $\mathrm{OH}$ would produce enough $\mathrm{OH}$ to be observed. If a separate oxygen-rich shell exists, it appears that it has to be a high-density shell distinct from the carbon-rich molecular shell. Since multiple shells are known to exist in larger planetaries (Chu, Jacoby, \& Arendt 1987; Balick et al. 1992), this is not a totally unreasonable scenario. Until the water distribution in NGC 7027 can be mapped by high-resolution observations, we consider this possibility to be unlikely.

\subsubsection{Other Water Lines}

Figure 3 shows the model spectrum for the ortho$\mathrm{H}_{2} \mathrm{O} / \mathrm{CO}=0.00012$ case using a fixed line width of FWHM $0.7 \mu \mathrm{m}$. The total fluxes of the lines are summarized in Table 3. Figure 4 shows the simulated spectrum for a broader wavelength range with all the $\mathrm{CO}$ rotational lines included, along with the nebular lines and all calculated water lines. In this figure the line width is given by the expansion velocity of the nebula (FWHM $30.4 \mathrm{~km} \mathrm{~s}^{-1}$ ). All predicted molecular lines from $\mathrm{CO}$, ortho-water, and para-water are included. Some of the weaker water lines are in absorption.

The strengths of a number of other water lines within the ISO LWS band are also predicted from the model. Figure 4 shows the ortho lines at $108.1 \mu \mathrm{m}\left(2_{21} \rightarrow 1_{10}\right), 180.5 \mu \mathrm{m}$ line $\left(2_{21} \rightarrow 2_{12}\right) 174.6 \mu \mathrm{m}\left(3_{03} \rightarrow 2_{12}\right)$, and various other, weaker water lines. The 180.5 and $174.6 \mu \mathrm{m}$ lines (shown in Fig. 4 as the two weak lines to the right of the $\mathrm{H}_{2} \mathrm{O} 2_{12}-1_{01}$ and the CO 15-14 lines, respectively) are predicted to have less than half of the strength of the $179.5 \mu \mathrm{m}$ line for the range of water abundances we have covered in our calculations, and may not be detectable in the existing ISO LWS spectrum of NGC 7027. The $108.1 \mu \mathrm{m}$ line is found in the model to be strongly dependent on the water abundance, with a strength of only $15 \%$ of the $179.5 \mu \mathrm{m}$ line in the best-fit model but having 3 times the strength of the $179.5 \mu \mathrm{m}$ line at the largest abundance ratio. This line is thus a more sensitive measure of the water abundance than any of the other accessible water lines. This line might be blended with the $\mathrm{CO} 24 \rightarrow 23$ line at $108.76 \mu \mathrm{m}$, although the $\mathrm{CO}$ lines below $120 \mu \mathrm{m}$ are predicted to be weak in our model.

Since the $108.1 \mu \mathrm{m}$ line requires higher temperatures to excite, it originates mainly from the shell. Our models suggest that the $108.1 \mu \mathrm{m}$ line will be stronger than the

TABLE 3

EXamples of Predicted INFRARed Line FluXes

\begin{tabular}{cccccc}
\hline \hline LINE & C II & CO (14-13) & CO (15-14) & CO (16-15) & $\mathrm{H}_{2} \mathrm{O}\left(2_{12}-1_{01}\right)$ \\
\hline Wavelength $(\mu \mathrm{m}) \ldots \ldots \ldots$ & 157.7 & 186.0 & 173.6 & 162.8 & 179.5 \\
Flux $\left(\mathrm{ergs} / \mathrm{cm}^{-2} \mathrm{~s}^{-1}\right) \ldots \ldots$ & $5.9 \times 10^{-12}$ & $8.8 \times 10^{-13}$ & $9.6 \times 10^{-13}$ & $9.5 \times 10^{-13}$ & $5.5 \times 10^{-13}$ \\
\hline
\end{tabular}




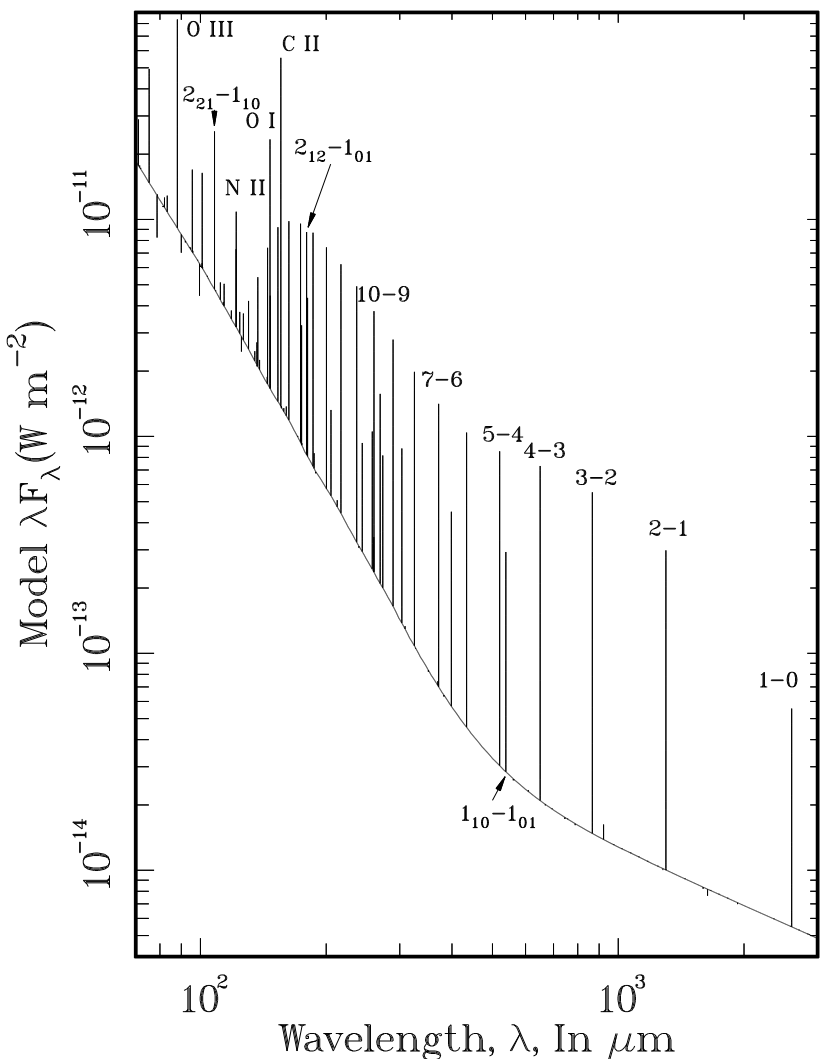

FIG. 4.-Model far-infrared spectrum of NGC 7027 showing the CO rotational lines, the nebular lines, and the water lines. The lines are plotted as Gaussians of widths determined by the expansion speed of the nebula. This simulated spectrum assumes a constant, uniform beam independent of frequency.

$179.5 \mu \mathrm{m}$ line as long as water is present in the inner $20^{\prime \prime}$ of the envelope. The measurement of the ratio of these two lines would be useful in determining the exact distribution of the water molecule in the neutral envelope.

We have also calculated the strength of the $556.945 \mathrm{GHz}$ water line as it would be observed with a beam size comparable to that of the Odin satellite. The Odin line is predicted to have a brightness temperature of $0.037 \mathrm{~K}$ with a normal, optically thick, flat-top profile (total line strength $0.78 \mathrm{~km}$ $\left.\mathrm{s}^{-1}\right)$. Due to the large Odin beam $\left(\sim 2^{\prime}\right.$ at this frequency), the effect of beam dilution is severe for NGC 7027. While this predicted line strength varies with the $\mathrm{H}_{2} \mathrm{O} / \mathrm{CO}$ abundance ratio, the dependence is not strong since the line is optically thick.

\section{THE SPECTRUM OF NGC 7027}

The combined model results, representing the entire spectrum of NGC 7027, are plotted in Figure 5. The heights of the individual spectral lines are calculated from the total line strength assuming a $\Delta \lambda / \lambda$ of 0.00125 . In the mid- and far-infrared, the continuum emission is dominated by the dust component. The major PAH features at 3.3, 7.7, 8.6, and $11.3 \mu \mathrm{m}$ can be seen as narrow features. At wavelengths longer than $\sim 400 \mu \mathrm{m}$, the $\mathrm{ff}$ continuum begins to be stronger than the dust continuum. The visible and nearinfrared continua are marked by the hydrogen and helium bf jumps. The broad feature near $2400 \AA$ is the $2-\gamma$ continuum. The absorption feature seen in the near-UV is the $2200 \AA$ feature. The blackbody spectrum of the central star

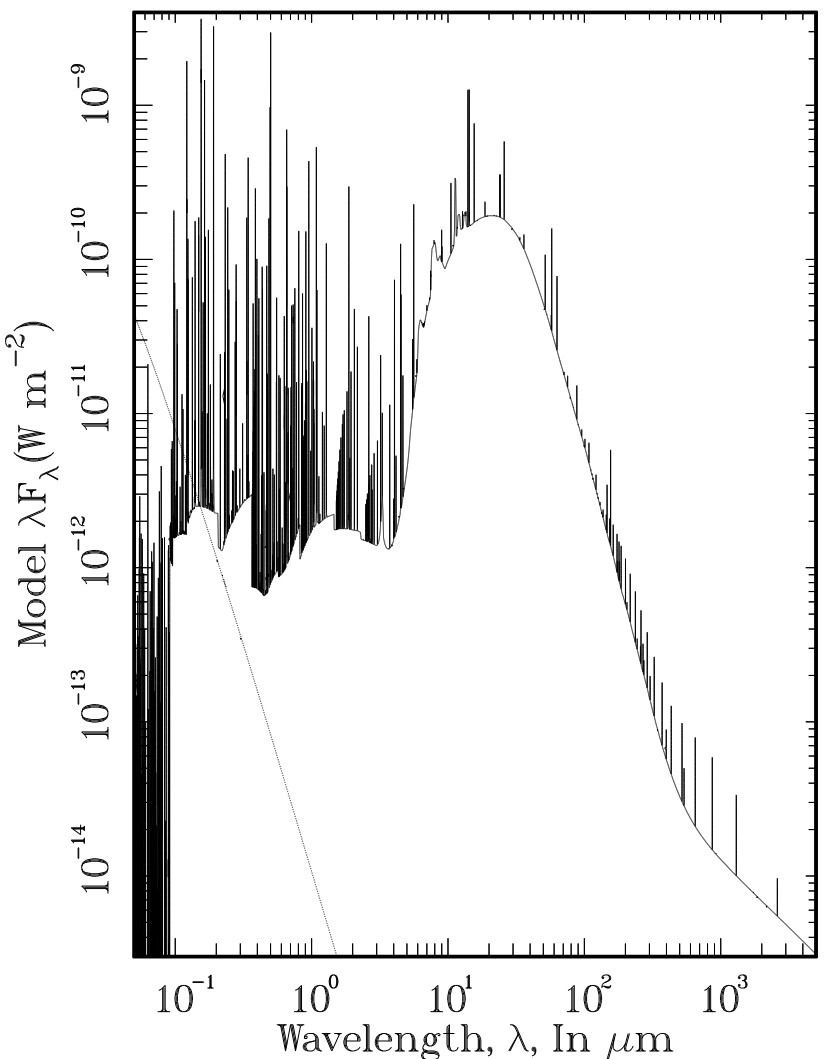

FIG. 5.-The full combined-model spectrum for NGC 7027 including all the nebular emission lines, the dust emission, and the molecular lines calculated in the model. The blackbody spectrum of the central star is shown as a dotted line.

is plotted as a dotted line. Since we assume that the ionized region is ionization bounded, all the Lyman continuum $(\lambda<912 \AA)$ photons are absorbed by the nebula. The emission spectrum beyond the Lyman limit is all nebular lines from higher ionization states of metals.

In order to compare the model spectrum with observations, we have reproduced the observed NGC 7027 spectrum from Terzian (1989) in Figure 6a, and a plot of the model spectrum in $F_{v}$ versus $v$ in Figure $6 b$. Although we have not tried to fit every detail of the observed spectrum, the overall resemblance of the two is striking.

\section{DISCUSSION}

\subsection{Water as a Fraction of the Total Oxygen Abundance}

Assuming that the $179.5 \mu \mathrm{m}$ water line has an observed strength of $0.61( \pm 10 \%)$ of that of the CO $14 \rightarrow 13$ line, the total water-to-hydrogen $\left[\mathrm{H}_{2} \mathrm{O} / \mathrm{H}_{2}\right]$ abundance is estimated to be from $2.22 \times 10^{-7}$ to $4.54 \times 10^{-7}$, with a nominal value of $3.17 \times 10^{-7}$. Emission-line analysis for NGC 7027 by Shields (1978) gave abundances of $0.0025_{-0.0009}^{+0.0015}$ for carbon and $0.00069_{-0.00014}^{+0.00018}$ for oxygen with respect to atomic hydrogen, or a $\mathrm{C} / \mathrm{O}$ ratio of 3.6. Values almost identical to these are found by Zuckerman \& Aller (1986) while Perinotto, Panagia, \& Benvenuti (1980) deduce values of 0.0013 and 0.00042 for the $\mathrm{C} / \mathrm{H}$ and $\mathrm{O} / \mathrm{H}$ number ratios, respectively. Middlemass (1990) finds abundance ratios of 0.00126 and 0.00055 , respectively. Whatever set of values is adopted, the ionized region has a $\mathrm{C} / \mathrm{O}$ ratio that is higher than average for planetary nebulae. The $\mathrm{CO} / \mathrm{H}_{2}$ number ratio of 0.0002 deduced by Jaminet et al. (1991) and used 


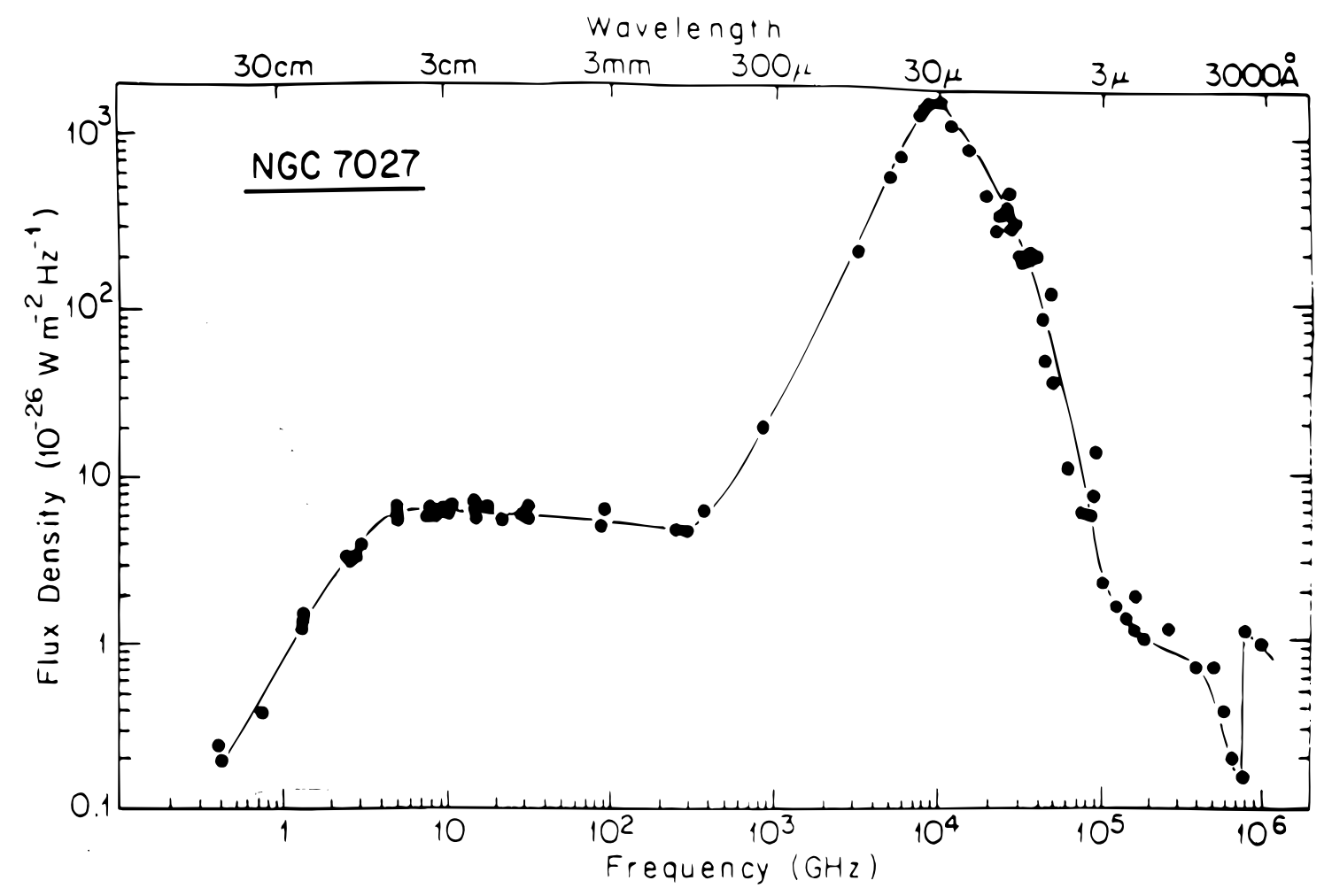

FIG. $6 a$

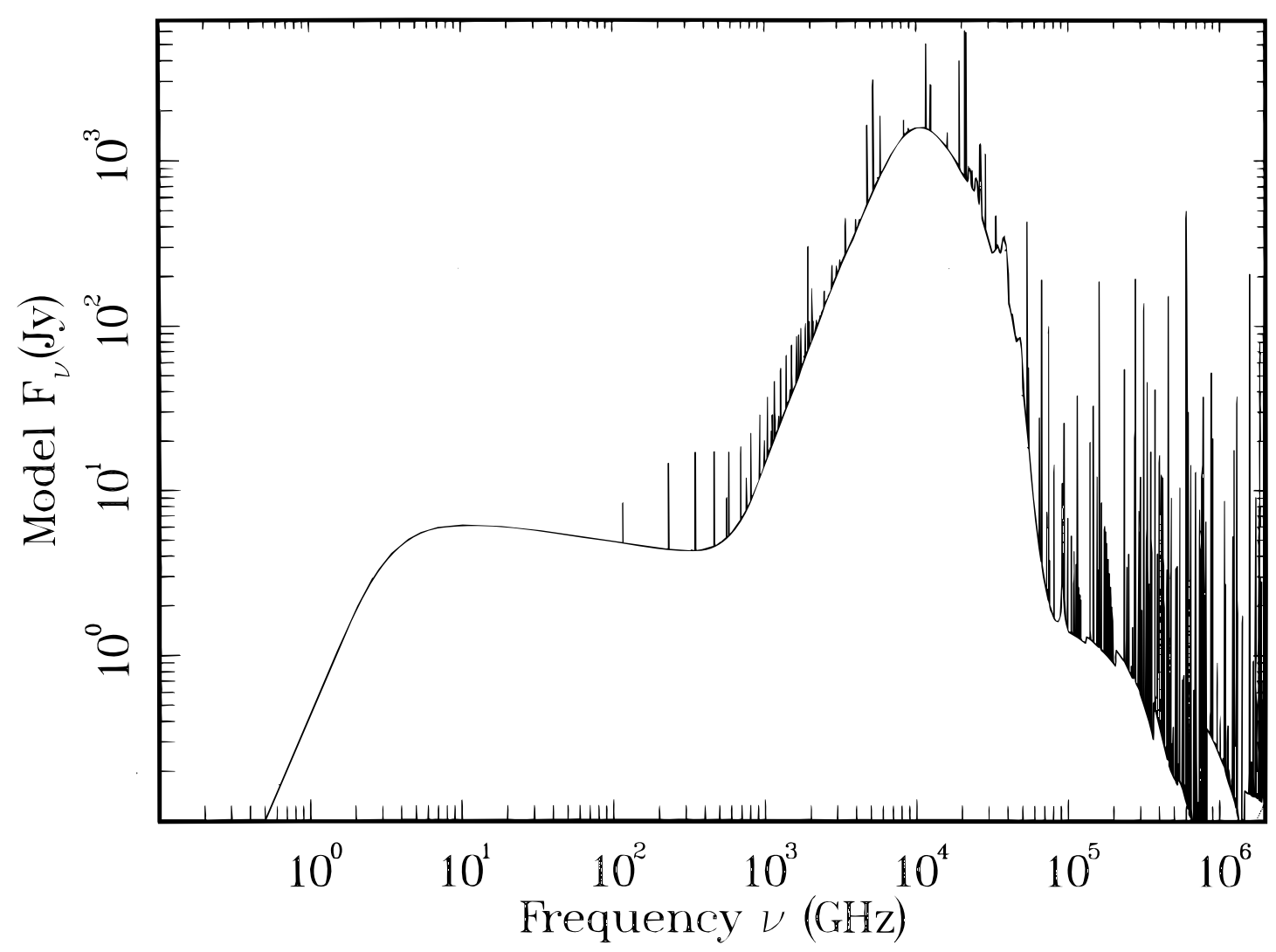

FiG. $6 b$

FIG. 6. (a) Observed spectrum of NGC 7027 adopted from Terzian (1989); (b) Model spectrum of NGC 7027 (same as Fig. 5) plotted as $F_{v}$ vs. $v$

here is lower than expected from the $\mathrm{O} / \mathrm{H}$ number ratios derived for the ionized region. The fraction of oxygen that has to be in the form of water is then $0.023_{-0.010}^{+0.018 \%}$ for the Shields oxygen abundance, $0.038_{-0.011}^{+0.016 \%}$ for the Perinotto oxygen abundance, or $0.029_{-0.009}^{+0.012 \%}$ for the Middlemass oxygen abundance.

Since the observed $\mathrm{CO}$ abundance represents only $15 \%-24 \%$ of the nebular oxygen abundance in NGC 7027 , 
the remaining oxygen in the molecular envelope may have been condensed onto grains. It is not clear whether there would be any observable effect of such ice mantles on the grains given the uncertainties in the grain properties for astronomical objects. However, it does not seem possible that a large part of the "missing" oxygen is either in the form of silicate grains or in the form of water ice. In the former case, the $9.7 \mu \mathrm{m}$ emission or absorption feature should be easily observable against the infrared continuum, as commonly seen in the spectra of $\mathrm{H}$ in regions. In the latter case, NGC 7027 should show the same infrared ice features at 44 and $62 \mu \mathrm{m}$ as seen in the Frosty Leo nebula (Omont et al. 1990).

Conventional chemical models predict that $\mathrm{CO}$ would be strongly favored over $\mathrm{H}_{2} \mathrm{O}$ in the carbon-rich conditions of the envelope of NGC 7027, so much so that the equilibrium models predict essentially no water to be present at all (McCabe, Connon Smith, \& Clegg 1979). Even under nonequilibrium chemical conditions, it is not certain that as much as $0.025 \%$ of the oxygen can be converted to water when the starting $\mathrm{C} / \mathrm{O}$ ratio is $\sim 2.5$. It is possible that the UV radiation from the nebula is responsible for the formation of water by causing photoionization of atoms and molecules and thus driving an active ion chemistry. If this is true, however, the water should be confined to the area around the photodissociation region, as the optical depth of the dust shell is large. While detailed models are required to assess this possibility, given the relatively small extent of the photodissociation region in the circumstellar shell of NGC 7027 it seems that this would not be able to make enough water to produce a column density sufficient to give a detectable line. Unless both oxygen-rich and carbon-rich chemistries are going on in the stellar wind at the same time (which would indicate that the dredge-up of carbon from the stellar core is strongly influenced by convection in three dimensions), the presence of water at this abundance implies that there the current chemical models are inadequate. A possible reason is that grain chemistry has not been properly accounted for. In order to test these possibilities, it would be interesting to see whether water molecules are also observed in carbon-rich AGB stars.

\subsection{How Accurate Is the Derived Water Abundance?}

In spite of the complexity of the model, there are relatively few free parameters. In order to reproduce the observed strength of the high- $J$ CO lines, a relatively warm and dense shell is needed to excite $\mathrm{CO}$ emission. The models suggest that the higher $\mathrm{CO}$ lines are entirely collisionally excited. If the line strengths can be measured sufficiently accurately, the shell kinetic temperature could be found very exactly. The physical conditions of the shell and the strength of the observed $179.5 \mu \mathrm{m}$ water line then determine the water abundance.

This is, however, not to say that the photoionization model and the dust radiative transfer model have no effect on the molecular line strengths. In order to illustrate that the dust continuum radiation field does have an effect on the molecular line strengths, we have carried out a molecu- lar line transfer model using only the $2.73 \mathrm{~K}$ cosmic background radiation field but no dust continuum. The 179.5 $\mu \mathrm{m}$ line is much weaker in this model, with a strength of only $62 \%$ of the value when the dust radiation field is included. The higher $\mathrm{CO}$ rotation lines are also slightly weaker, although it is only a $4 \%$ effect. This test demonstrates that stimulated absorption of dust continuum photons plays a significant role in the excitation of the water molecule. The omission of this effect would lead to an artificially high water abundance.

The derived water abundance is sensitive to the strength of the water line measured: an increase of the line strength by $67 \%$ requires more than a factor of 100 increase in water abundance (see Table 2). The next source of uncertainty is where in the circumstellar shell the water is located. If the water exists in only part of the neutral region, the abundance could be much higher than the abundance value estimated here.

\section{CONCLUSIONS}

The planetary nebula NGC 7027 is an interesting object to study because it produces strong emissions across the electromagnetic spectrum ranging from UV to radio. Extensive ground-based and space-based observations have produced a wealth of photometric and spectroscopic measurements that reflect a rich assembly of physical processes. In this paper, we have made a first attempt to model the entire nebular spectrum, taking into account most of the relevant emission mechanisms, and consistently treated the radiative couplings between the various (ionized, molecular, and dust) components. We have successfully reproduced the entire spectral energy distribution of the object in the wavelength range of $1000 \AA$ to $1 \mathrm{~cm}$. Specifically, we have fitted the recently observed far-infrared lines of $\mathrm{CO}$ and water in the $170 \mu \mathrm{m}$ region, and deduced the abundance ratio of $\mathrm{H}_{2} \mathrm{O} / \mathrm{H}_{2}$ as $3.2 \times 10^{-7}$.

This exercise has demonstrated the value of multiwavelength observations. Our model of NGC 7027 has also shown that there are intricate interconnections between the different components of the planetary nebula system. This advance was also made possible by the availability of highspeed computing facilities, for such attempts at combined continuum/line transfer problems were unthinkable just a decade ago. We hope that this paper serves only as the beginning of more extensive efforts in the complete solutions to the radiation problems in celestial objects, not only for planetary nebulae, but also for active galactic nuclei and other objects that show a variety of emission processes in their spectrum.

This work is supported by the Canadian Space Agency and by the Natural Sciences and Engineering Research Council of Canada. We thank G. Ferland, S. Deguchi, and C. M. Leung for the use of their computer codes. We also thank Mike Barlow for communication of the ISO results prior to publication.

\section{REFERENCES}

Balick, B., Gonzalez, G., Frank, A., \& Jacoby, G. 1992, ApJ, 392, 582 Bieging, J. H., Wilner, D., \& Thronson, H. A. 1991, ApJ, 379, 271

Bregman, J. D., Allamandola, L. J., Tielens, A. G. G. M., Geballe, T. R., \& Witteborn, F. C. 1989, ApJ, 344, 791

Chu, Y., Jacoby, G. H., \& Arendt, R. 1987, ApJS, 64, 529
Desert, F.-X., Boulanger, F., \& Puget, J. L. 1990, A\&A, 237, 215

Ferland, G. L. 1996, Hazy: A Brief Introduction to CLOUDY, Univ.

Kentucky Dept. of Phys. and Astron. Internal Rep.

Forrest, W. J., Houck, J. R., \& McCarthy, J. F. 1981, ApJ, 248, 195

Gillett, F. C., Forrest, W. J., \& Merrill, K. M. 1973, ApJ, 183, 87 
Gillett, F. C., Merrill, K. M., \& Stein, W. A. 1972, ApJ, 172, 367

Heap, S. R., \& Hintzen, P. 1990, ApJ, 353, 200

Hubeny, I., Heap, S. R., \& Lanz, T. 1994, BAAS, 185, 471

Hoare, M. G., Roche, P. F., \& Clegg, R. E. S. 1992, MNRAS, 258, 257

Jacoby, G. H. 1988, ApJ, 333, 193

Jaminet, P. A., et al. 1991, ApJ, 380, 461

Kwok, S. 1980, ApJ, 236, 592 1994, PASP, 106, 344

Leung, C. M. 1976, ApJ, 209, 75

Liu, X.-W., et al. 1996, A\&A, in press

Masson, C. 1986, ApJ, 302, L27 1989, ApJ, 336, 294

McCabe, E. M., Connon Smith, R., \& Clegg, R. E. S. 1979, Nature, 281, 263

McCarthy, J. F., Forrest, W. J., \& Houck, J. R. 1978, ApJ, 224, 109

Middlemass, D. 1990, MNRAS, 244, 294

Mufson, S. J., Lyon, J., \& Marionni, P. A. 1975, ApJ, 201, L85

Omont, A., et al. 1990, ApJ, 355, L27
Perinotto, M., Panagia, N., \& Benvenuti, P. 1980, A\&A, 85, 332

Pottasch, S. R. 1984, Planetary Nebulae (Dordrecht: Reidel)

Sandell, G. 1994, MNRAS, 271, 75

Schutte, W. A., Tielens, A. G. G. M., \& Allamandola, L. J. 1993, ApJ, 415, 397

Scott, P. F. 1973, MNRAS, 161, 35P

Shields, G. A. 1978, ApJ, 219, 565

Telesco, C. M., \& Harper, D. A. 1977, ApJ, 211, 475

Terzian, Y. 1989, in IAU Symp. 131, Planetary Nebulae, ed. S. TorresPeimbert (Dordrecht: Kluwer), 17

Terzian, Y., Balick, B., \& Bignell, C. 1974, ApJ, 188, 257

Walton, N. A., Pottasch, S. R., Reay, N. K., \& Spoelstra, T. 1989, in IAU Symp. 131, Planetary Nebulae, ed. S. Torres-Peimbert (Dordrecht: Kluwer), 301

Woolf, N. J. 1969, ApJ, 157, L37

Zuckerman, B., \& Aller, L. H. 1986, ApJ, 301, 772 